\title{
SPATIAL AND TEMPORAL CHANGE ANALYSIS OF URBAN HEAT ISLAND EFFECT IN WUHAN CITY
}

\author{
Huang Minhui,Chen Jianjun* \\ College of Geomatics and Geoinformation, Guilin University of Technology, Guilin 541004, China \\ 870409694@qq.com(H.M); chenjj@glut.edu.cn(C.J)
}

KEY WORDS: Urban Heat Island; driving factor; trend analysis

\begin{abstract}
China has experienced rapid urbanization and rapid development of economy in the past decades, resulting in severe damage to the urban ecological environment, causing changes in the urban thermal environment and triggering the urban heat island effect. Moreover, the heat island effect has become a hot topic for scholars. The urban heat island effect refers to the phenomenon that the urban surface temperature is significantly higher than that of surrounding suburbs due to the interaction of man-made and natural. The city is considered to be the largest man-made ecosystem. Its heat island effect will not only change the growth habit of urban vegetation, but also affect the outer environment of urban buildings, it further influences human life and has a great negative impact on human health. Therefore, the study of the spatial-temporal variation characteristics of urban heat island effect and its influencing factors can provide data support for the environmental quality control and urban planning of local government departments. Based on the surface temperature remote sensing product data, we studied the spatial distribution characteristics of urban heat island effect in Wuhan from 2001 to 2013, by calculating the temperature difference between the highest and lowest temperatures and the average interval method for heat island classification. We conducted a trend analysis of vegetation cover from 2001 to 2013 initially explore the effects of vegetation cover $n$ heat island effect. The results showed that: (1) From 2001 to 2013, the intensity of heat island in Wuhan was strong in the city center, weaker surrounding city center and the weakest in the suburbs; From 2001 to 2011, the intensity of heat island in Wuhan city was significantly weaken, among which Huangpi, Xinzhou, Jiangxia, Hannan and Caidian district were weaken, and the urban heat island effect of the city center was enhanced; From 2011 to 2013, the intensity of heat island in Wuhan city presented an increasing trend, among which Huangpi district, Xinzhou district and Caidian district were the most obvious, and the urban heat island effect was slightly weaken. (2) Between 2001 and 2013, the vegetation cover in Huangpi district and Xinzhou district increased significantly, and the vegetation cover in the downtown, Jiangxia district and Dongxihu district decreased significantly, corresponding to the urban heat island effect of Wuhan increased volatility. Our results showed that the spatial distribution of urban heat island effect in Wuhan city fluctuated with time during the study period, and the vegetation cover had a significant influence on it..
\end{abstract}

\section{INTRODUCTION}

Urban heat island effect refers to the phenomenon that the temperature of urban area is obviously higher than that of surrounding suburbs under the combined action of human and nature.Because the suburban near-surface temperature changes slightly, while the urban near-surface temperature increases significantly, like a small island in the middle of the sea, it is called the urban heat island ${ }^{[1]}$. As the most obvious product of urbanized environmental pollution, the heat island effect changes the thermal structure of urban underlying surface, making the environmental temperature value in the spatial model of temperature higher ${ }^{[2]}$. With the accelerated development of urbanization economy,this heat island effect will

${ }^{*}$ Corresponding author 
become more and more obvious, which will change the growth habits of urban vegetation, local climate ${ }^{[3]}$, image air quality ${ }^{[4]}$, physical and chemical properties of soil ${ }^{[5]}$ and many other urban ecological processes.As one of the driving factors affecting the heat island effect, the change of the way, type and degree of urban greening construction will lead to the continuous appearance of negative environmental effects such as surface temperature rise and heat island effect.For a long time, scholars have used meteorology, remote sensing, numerical simulation and other research methods to study the heat island effect,Alonso et al used meteorology to analyze the urban heat island effect in Salamanca, Spain, under different weather types ${ }^{[6]}$,In 2013, when Manju Mohan and other scholars used remote sensing data to retrieve the surface temperature of New Delhi, they found that the high temperature centers of urban heat island effect usually appeared in commercial centers and buildings ${ }^{[7]}, \mathrm{Hu}$ Xiaoming et al. simulated the boundary layer characteristics of Beijing on July 10 and 11, 2000 using a non-hydrostatic equilibrium moderate regime model $^{[8]}$.

At present, many scholars focus their research on heat island effect on remote sensing data.According to the consistent correlation between surface temperature and meteorological temperature, the heat island grade is divided by normalizing the surface temperature, and the heat island grade is analyzed.Then, by means of the inversion algorithm of surface temperature, the surface temperature of a certain area can be accurately divided.It is more convenient and accurate than other research methods. This paper is based on MODIS LST data and MODIS NDVI data of Wuhan from 2002 to 2009,Using equal interval method to classify heat island, the spatial distribution pattern and temporal variation characteristics of surface temperature were studied.Combined with the influence of vegetation coverage on surface temperature, it provides scientific reference for future urban planning and construction in Wuhan.

\section{MATERIALS AND METHODS}

\subsection{Survey of Research Areas}

Wuhan is located in the eastern part of Jianghan Plain and the middle reaches of Yangtze River. Its longitude and latitude range is $113^{\circ} 41^{\prime}-115^{\circ} 05^{\prime}$ in the East and $29^{\circ} 58^{\prime}-31^{\circ} 22^{\prime}$ in the north,the climate belongs to subtropical monsoon climate. It has the characteristics of warm spring, cool autumn, hot summer and cold winter, concentrated rainwater in summer, dispersed winter and abundant water resources. Wuhan is located in the transitional area from the evergreen broad-leaved forest in the middle subtropical zone to the deciduous broad-leaved forest in the north subtropical zone. The vegetation of the whole city has floristic elements in both the South and the north,the mixed forest composed of evergreen broad-leaved forest and deciduous broad-leaved forest is a typical vegetation type in the city.

As a central city in central China, Wuhan is the core city of the Yangtze River economic belt, an important industrial base, a science and education base and a comprehensive transportation hub.Because of its unique geographical location, Wuhan is also the largest land, water and air transport hub in China's inland. The permanent population of cities and towns reaches 8.89 million people throughout the year, and the humanities and economy develop rapidly.

\subsection{Data Sources and Data Processing}

2.2.1 Data Sources:The MODIS LST data used in this paper are derived from the MYD11A2 LST product data provided by the geospatial data cloud (http://www.gscloud.cn/) from July 2002 to 2009. The row and column number of MODIS is H27V05.The spatial resolution of the data is 1000 meters, the temporal resolution is 8 days, the data format is Hdf, and the map projection is Sinusoidal (sinusoidal curve projection).MODIS vegetation data are derived from MODIS product data provided by geospatial data cloud (http://www.gscloud.cn/) from 2002 to July 2009. The row number of MODIS is H27V05.The 
spatial resolution of the data is 1000 meters, the temporal resolution is 16 days, the data format is $\mathrm{Hdf}$, and the map projection is Sinusoidal (sinusoidal curve projection)

2.2.2 Data Processing:Data Preprocessing Using MRT (MODIS Reprojection Tools) Projection Tools, The spatial resolution of the original MYD11A2 data is $930 \mathrm{~m}$, and the data is resampled by the method of near nature, which makes the data standard $1000 \mathrm{~m}$, The original format is converted to Geotiff, and the original projection is converted to Albertian Graded Conical Projection,finally, the data are joined together.In ENVI, the MYD11A2 data set after re-projection is clipped with the vector boundary of Wuhan as the mask.

\subsection{Research methods}

According to the time sequence of the administrative division of Wuhan, this paper chooses Jiangan District, Qingshan District, Jianghan District, Qiekou District, Wuchang District, Hanyang District and Hongshan District as the central urban area (Fig. 1), and the rest as the suburban area.

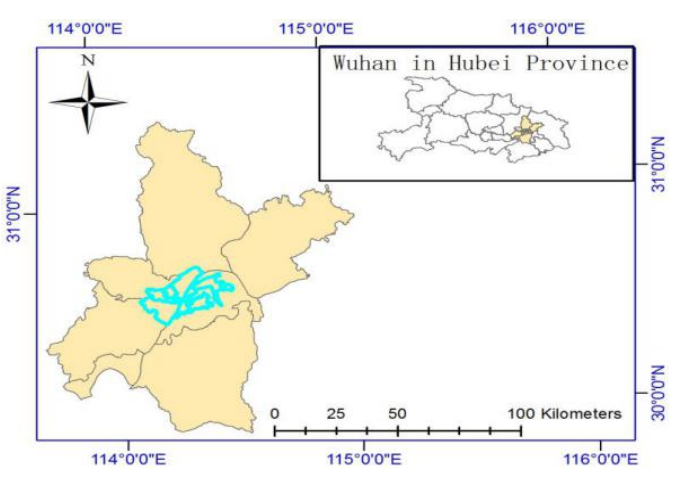

Fig. 1 Selection of urban area in Wuhan

In this paper, we use split window algorithm to retrieve the average LST products for 8 consecutive days from 2002 to 2009. In ARCGIS, we use grid calculator to extract the surface temperature, and then use equal interval method to classify the heat island intensity,vegetation coverage was estimated by pixel dichotomy model,and the effects of spatial distribution pattern and temporal variation characteristics of vegetation coverage on surface temperature in Wuhan were studied.

\section{RESULTS AND ANALYSIS}

\subsection{Spatial and Temporal Changes of Urban Heat Island Effect in Wuhan}

Referring to the relevant literature and the actual situation of surface temperature in Wuhan, the method of dividing surface heat field by equal interval is used to divide the surface temperature in July of 2002-2009 in Wuhan.After normalization, the surface temperature was divided into five levels, namely, low temperature zone $(0<\mathrm{UHI}<0.2)$, medium low temperature zone $(0.2<\mathrm{UHI}<0.4)$, medium temperature zone $(0.4<\mathrm{UHI}<0.6)$, sub-high temperature zone $(0.6<\mathrm{UHI}<0.8)$, high temperature zone $(0.8<\mathrm{UHI}<1)$. When the surface temperature field is divided into five levels, it is generally considered that the high temperature area and sub-high temperature area can represent the urban heat island area[9]. Therefore, in this paper, the high temperature area and sub-high temperature area are divided as the heat island area.

The heat island area classification map of July in Wuhan is superimposed on the vector map of the administrative region of Wuhan, as shown in Figure 2 ,Red is the high temperature area and orange is the sub-high temperature area. The combination of the two is the heat island area of the study area.From 2002 to 2009, the area of heat island in Wuhan expanded in fluctuation, spreading from the central urban area to the surrounding areas,and there are heat islands in seven districts in the center of Wuhan city.From 2002 to 2004, the area of heat island in Wuhan was mainly concentrated in several areas in the city center.By 2005, the area of heat island began to appear in sheet form in Jiangxia, Huangpi and East-West Lake areas.By 2006, the heat island area was mainly concentrated in the eight districts in the city center, Huangpi district, Xinzhou district and East-West Lake district.In 2007, the area of the heat island decreased, mainly concentrated in the city centre, and appeared in Huangpi District and Huangpi 
District in discontinuous small areas.In 2008, the area of heat island increased again, mainly distributed in eight districts of Shizhong District, East-West Lake District, Caidian District, northern Xinzhou District and Northern Huangpi District, sporadically distributed in other districts.By 2009, the area of heat island decreased, mainly concentrated in eight
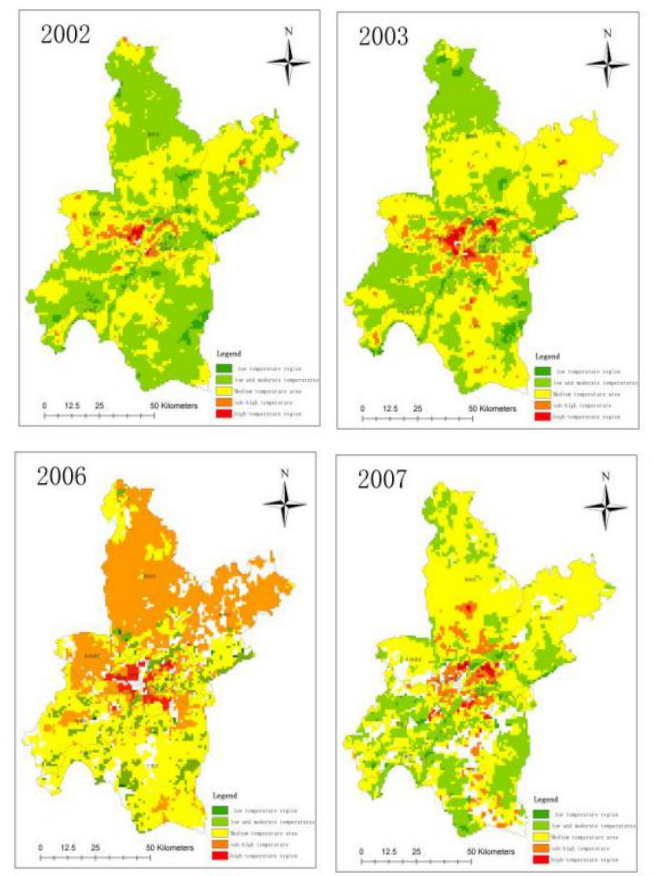

districts in the city center, Jiangxia district, and a small number of flake heat island districts in Caidian district and Hannan district.On the whole, the heat island area of Wuhan tends to be concentrated and distributed in the central part of the city. There are a large number of heat islands in other areas in some years, and a small number in other years.
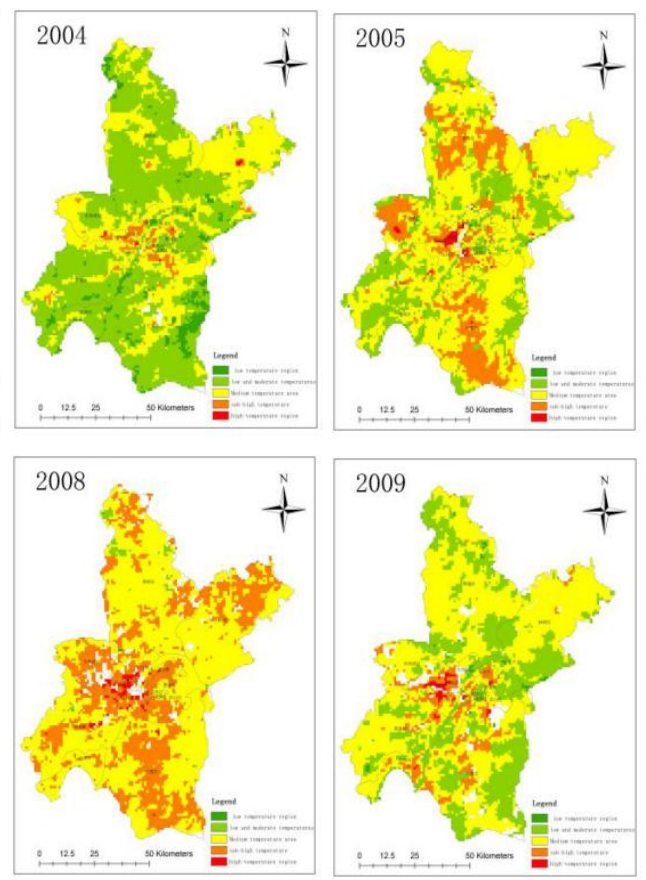

Fig. 2 Distribution Map of July Heat Island Area in Wuhan City

In this paper, the maximum heat island intensity method is used to evaluate the heat island effect in Wuhan. The maximum heat island intensity is the difference between the maximum temperature in the urban area and the minimum temperature in the suburbs, that

$$
P=T_{M A X}-T_{M I N}
$$

In the formula, $\mathrm{P}$ represents the maximum heat island intensity, $\mathrm{T}_{\mathrm{MAX}}$ represents the highest surface temperature, and $\mathrm{T}_{\mathrm{MIN}}$ represents the lowest surface temperature $^{[9] \text {. }}$

As can be seen from the table, from 2002 to 2009, the maximum heat island intensity of Wuhan in July was in 2008, which was 31.78 , and the smallest was in 2002, which was 17.42.The variation of maximum heat island strength is shown in Figure 3.

\begin{tabular}{cc}
\hline Year & Temperature \\
\hline 2002 & 17.42 \\
2003 & 19.56 \\
2004 & 19.02 \\
2005 & 26.32 \\
2006 & 24 \\
2007 & 18.12 \\
2008 & 31.78 \\
2009 & 27.9 \\
\hline
\end{tabular}

Table 1 Statistics of the Maximum Heat Island Intensity in July in Wuhan City 


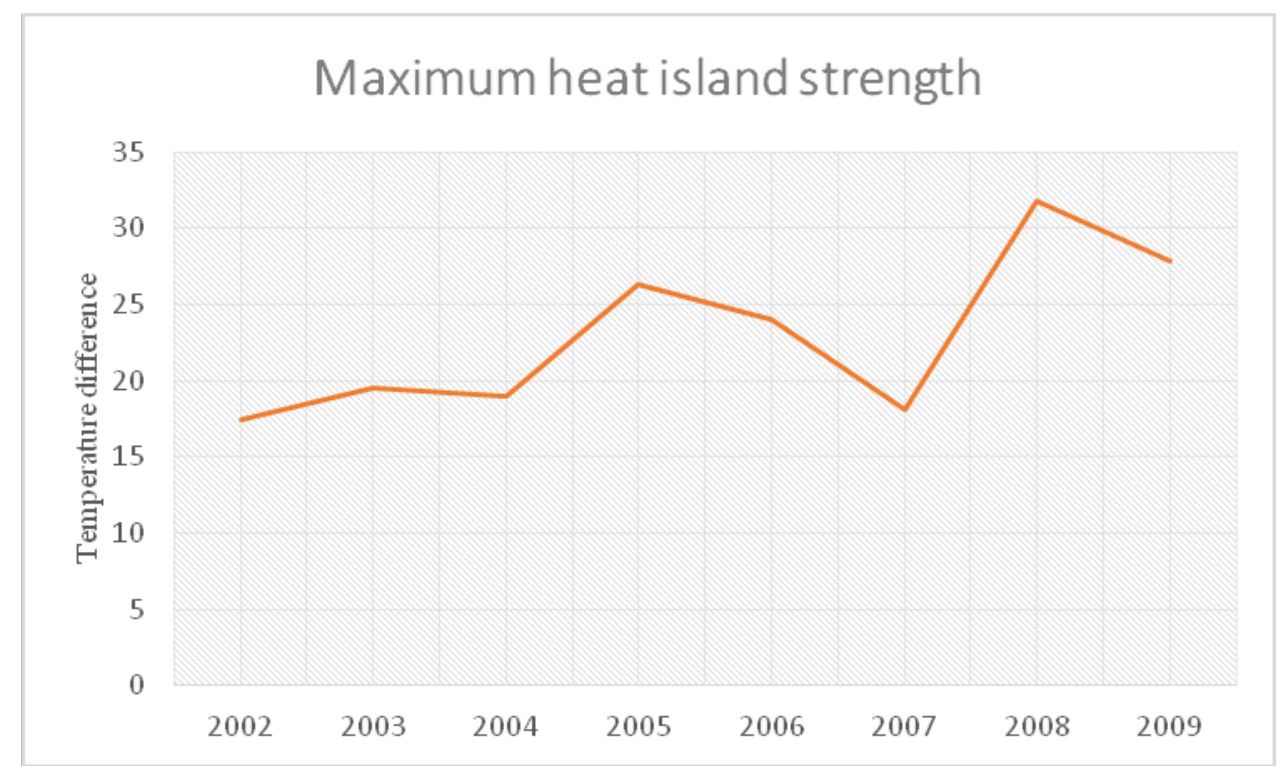

Fig. 3 Trend Chart of July Maximum Heat Island Intensity in Wuhan City

\subsection{Effects of vegetation on heat island effect in Wuhan}

Referring to the standard for classification and gradation of soil erosion ${ }^{[9]}$, and the actual vegetation cover in Wuhan,the vegetation cover is divided into five grades, Low vegetation coverage,Medium and low coverage,Medium coverage,Medium and high coverage, and High coverage.The classification is shown in Figure.4.The dark red in the picture above is water, and the light red indicates low vegetation coverage,Pink represents medium and low vegetation coverage, gray green represents medium vegetation coverage, light green represents medium and high vegetation coverage, dark green represents high vegetation coverage.

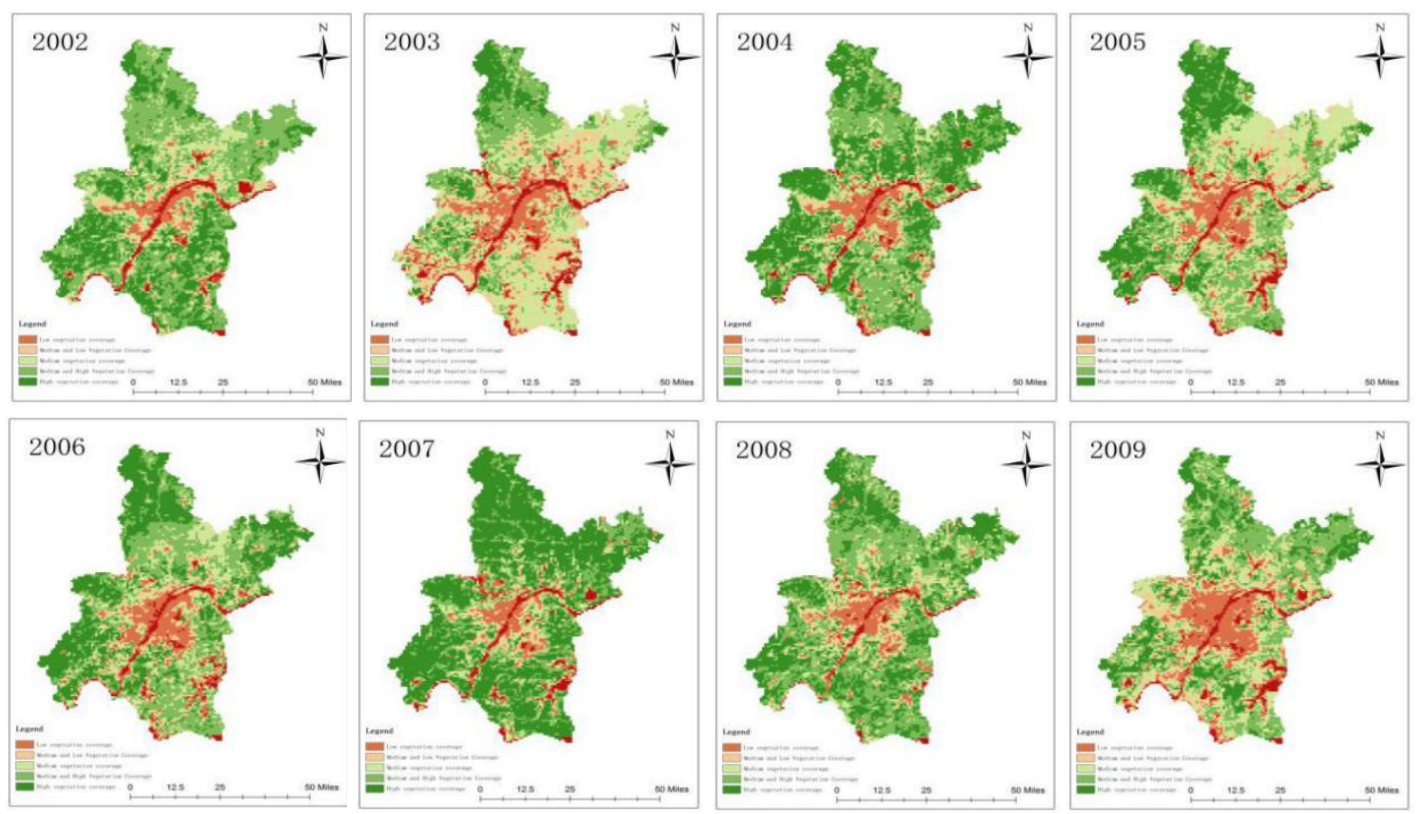

Fig.4 Classification of vegetation coverage in Wuhan

(Low vegetation coverage $<=50 \% ; 50 \%<=$ Medium and low coverage $<=65 \% ; 65 \%<=$ Medium coverage $<=80 \% ; 80 \%<=$ Medium and high coverage $<=95 \%$; High coverage $>=95 \%$ ) 
Low vegetation coverage mainly concentrated in the urban area of Wuhan, scattered or discontinuous patches in several other districts.Low and medium vegetation coverage mainly distributes in the edge of urban areas, sporadically in other regions.Medium vegetation coverage and medium and high vegetation coverage mainly distribute in the suburbs in a continuous area, while high vegetation coverage distributes in a large number of discontinuous patches in the suburbs.The variation characteristics of vegetation coverage over time in Wuhan City are obtained by statistical analysis of the coverage areas of vegetation coverage at all levels in Wuhan City as shown in Fig. 5.From 2002 to 2009, the area of low vegetation coverage in Wuhan showed fluctuating growth, and concentrated in the urban area of Wuhan, covering the surrounding areas of the city.Medium and low vegetation coverage in Wuhan also showed fluctuating growth, and distributed discontinuously in all districts of the city.The growth trend of medium vegetation coverage is consistent with that of low vegetation coverage and medium and low vegetation coverage.From 2002 to 2009, the middle and high vegetation coverage and the high vegetation coverage area showed a fluctuating decrease, mainly in the area around the city center.

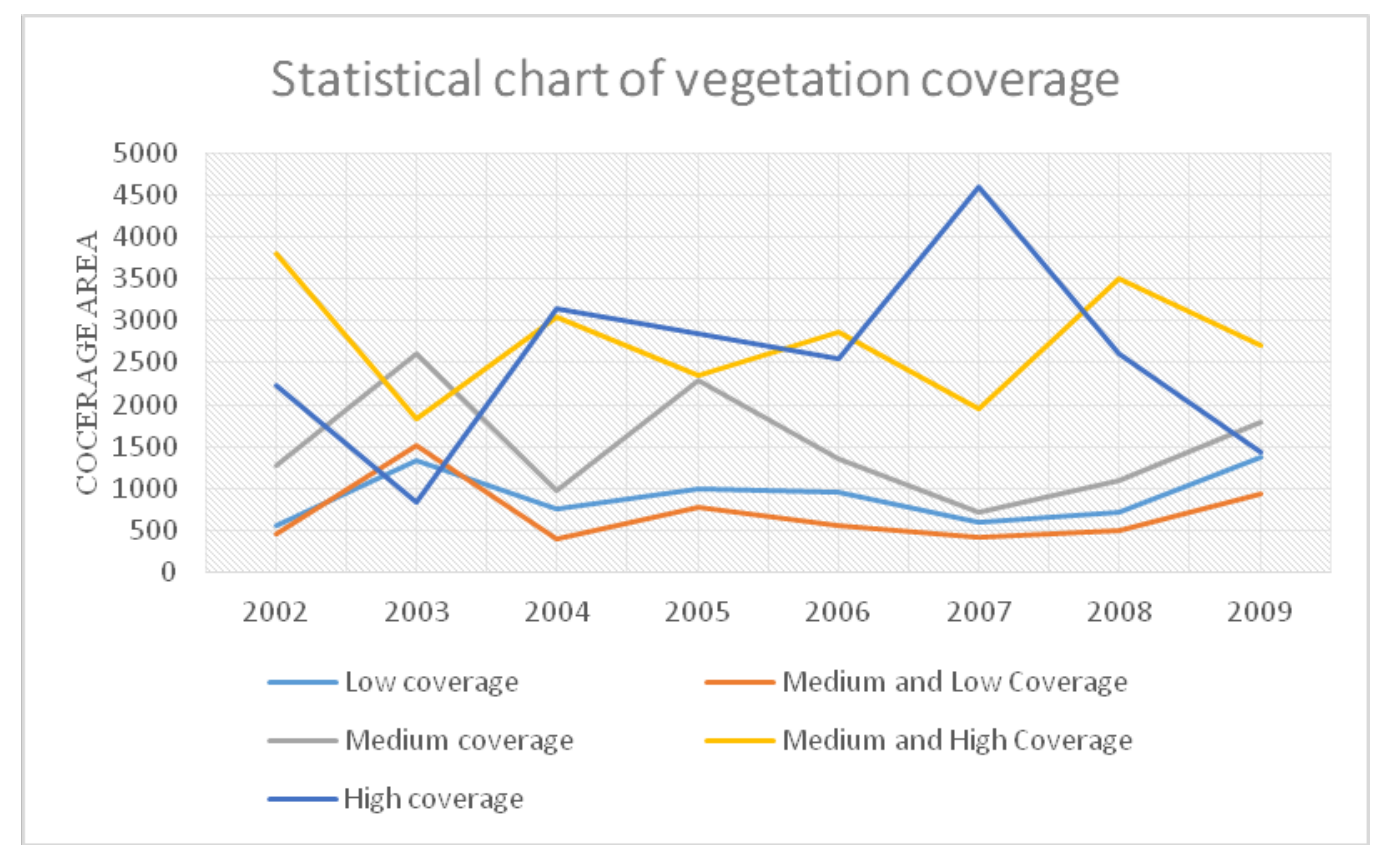

Fig. 5 Change characteristics of vegetation coverage in Wuhan from 2002 to 2009

From 2002 to 2003, the area of high vegetation coverage and medium and high vegetation coverage decreased significantly in Wuhan,medium vegetation coverage, medium and low vegetation coverage and low vegetation coverage increased significantly.It is consistent with the trend of decreasing the low temperature zone, increasing the high temperature zone and increasing the maximum heat island intensity in Wuhan from 2002 to 2003.By 2004, the growth of medium and high vegetation coverage and high vegetation coverage was consistent with the trend that the low and medium temperature areas increased, the high temperature areas decreased and the maximum heat island intensity weakened in
Wuhan in that year.In 2005, the area of high vegetation coverage and medium-high vegetation coverage decreased, the area of medium vegetation coverage increased sharply, the coverage of medium-low vegetation and low vegetation increased slightly, which was consistent with the trend of decreasing the low-temperature area, increasing the medium-high temperature area and increasing the maximum heat island intensity in Wuhan in 2005.In 2006, the area of high vegetation coverage, medium vegetation coverage and medium and low vegetation coverage decreased, and the area of medium and high vegetation coverage increased, which was consistent with the trend of small expansion of low and 
medium-low temperature areas in Wuhan in 2006 and the weakening of maximum heat island intensity.In 2007, the area of high vegetation coverage in Wuhan increased substantially, which accords with the phenomena that the sub-high temperature area decreased, the medium-low temperature area increased and the maximum heat island intensity decreased significantly in 2007.In 2008, the area of high vegetation coverage decreased sharply, the area of low vegetation coverage and medium and low vegetation coverage increased, which accords with the phenomenon that the intensity of the largest heat island in Wuhan increased sharply in 2008.In 2009, the area of high vegetation coverage continued to decrease, but the area of medium and high vegetation coverage increased, which was consistent with the distribution of heat island in Wuhan in 2009. On the whole, the temporal variation characteristics of vegetation coverage in Wuhan were basically consistent with the temporal variation characteristics of heat island intensity in Wuhan.

\section{CONCLUSION}

Based on MODIS synthetic products, this paper studies and analyses the spatial distribution characteristics, temporal variation characteristics of urban heat island intensity in Wuhan and the influence of vegetation coverage on it.Through the above research, we can draw the following conclusions:

(1) The distribution of surface temperature field in Wuhan is mainly in the middle-temperature zone, followed by the middle-high temperature zone or the middle-low temperature zone.Evolution between each temperature region is usually gradual,the evolution direction of heat island enhancement is as follows,Low Temperature Zone $\rightarrow$ Medium and Low Temperature Zone $\rightarrow$ Medium Temperature Zone $\rightarrow$ Medium and High Temperature Zone $\rightarrow$ High Temperature Zone, on the contrary, the direction of heat island weakening.It's also possible to evolve across levels, but less likely.

(2) From 2002 to 2009, the area of high-temperature and sub-high-temperature zones in Wuhan showed fluctuating growth,The maximum heat island intensity also shows an increase in fluctuation.Therefore, the heat island effect of Wuhan has an increasing trend in the future.

(3) The vegetation coverage in Wuhan mainly consists of medium and high vegetation coverage and high vegetation coverage,the distribution of heat island is consistent with that of Wuhan,the characteristics of time variation are basically consistent with those of heat island intensity in Wuhan.It can be seen that vegetation coverage has an important influence on the change of heat island intensity,reasonable increase of vegetation cover helps to reduce urban heat island effect.

(4) In this paper, the relationship between vegetation cover and heat island intensity is studied. It is found that heat island intensity and vegetation cover may have bidirectional effects, and there may be other factors affecting the change of heat island intensity.Therefore, in the future study, more driving factors of heat island intensity will be further studied.

\section{ACKNOWLEDGEMENTS}

This study was supported by the National Natural Science Foundation of China (41801030, 41901370, 41961065); Guangxi Natural Science Foundation (2018GXNSFBA281054, 2018GXNSFBA281075, 2017GXNSFDA198016); Research Foundation of Guilin University of Technology (GUTQDJJ2017069); the BaGuiScholars program of the provincial government of Guangxi (Guoqing Zhou).

\section{REFERENCES}

Bai, Y., Wang, X., Jiang, H., et al, 2013: Urban Heat Island Effect Research Day. Journal of Meteorological Environment 29(2), 101-106.

Xiao, R., OuYang, Z., Li, W., et al, 2005: Ecoenvironmental effects of urban heat island. Journal of Ecology 25(8), 2055-2060.

Sun, J., Shu, W., 2007: Study on the influence of urban heat island effect on winter and summer precipitation in Beijing,Atmospheric Science 31(2), 
311-320.

Chen, S., Fang, W., Fang, X., et al, 2012: Impact of urban heat island effect characteristics on air pollution in Zhumadian. Forum of the 29th Annual Meeting of China Meteorological Society, Shenyang, China Meteorological Society, 6.

Luo, Q., 2010: Urban Eco-environmental Construc

-tion and Urban Heat Island Effect in Beijing.

Collected Papers of the Symposium on"Beijing

Landscape Greening"2010, Beijing Landscape Society 5.

Alonso M S, Salazar J L, Fidalgo M R, et al., 2003:

Characteristics of the urban heat island in the city of

Salamanca, Spain. Atmosfera, 16(3), 137-148.

Mohan M, Kikegawa Y, Gurjar B R, et al, 2013:

Assessment of urban heat island effect for different land use-land cover from micrometeorological measurements and remote sensing data for megacity Delhi.Theoretical and Applied Climatology 112(3-4), 647-658.

Hu, X., Liu, S., Liang, F., et al, 2005: Numerical Simulation of the Characteristics of Near-Earth Boundary Layer in Beijing Region. Journal of Peking University(Natural Science Edition) 41(4), 514-522.

Xue, W., Lu, Z., Dang, S., et al, 2012: Urban Heat Island Effect Evaluation of Neijiang City, Sichuan Province based on Thermal Infrared Remote Sensing. Mapping and Spatial Geographic Information 35(4), $38-41$. 